\title{
Modified DSR Algorithm with MPR Selection Criteria
}

\author{
Priya Chakraborty \\ Mtech (CSE) \\ MRIU, Faridabad, India
}

\author{
Indu Kashyap, Ph.D \\ Associate professor \\ MRIU, Faridabad, India
}

\begin{abstract}
MANET is a collection of wireless mobile devices that communicate with each other without the use of any wireline network. In MANET due to its dynamic topology each device is free to move in any direction. MANET have some issues like flooding or broadcast storm problem. These problems arise because of its dynamic topology, broadcasting characteristic and mobility. In this paper different types of broadcasting techniques like probabilistic, cluster based, area based in MANET had been studied, and have analyzed the broadcast storm problem. In this paper a solution for" storm problem" in reactive routing protocol by reducing rebroadcast messages have been proposed. The above mentioned problem have been catered by modifying the data structrure of route request message and have included MPR field .A modified algorithm M-DSR have been proposed that helps in minimizing the control overhead in reactive routing protocol .In this M-DSR data structure of message have been taken i.e BROADCAST MSG and M-RREQ MSG, that will further limit the no of control msg in the network which will increase the protocol performances.
\end{abstract}

\section{Keywords \\ MANET, MPR, DSR, FLOODING}

\section{INTRODUCTION}

MANET is a collection of mobile nodes forming a dynamic topology without any wired structure. Each mobile nodes act as router and helps in forwarding the information. MANET have dynamic network topology i.e. devices form their own network and use multiple hop routing to transmit information from one device to another. The main advantage of MANET is being wireless, mobile and flexible method of establishing communication in situations where it is not possible to create wired network or where there is absence of any infrastructure. It is useful in areas like battlefield, military and other emergency and disaster situations. Two types of network is described in MANET i.e infrastructured and infrastructureless. Routing is the act of moving information across an internetwork from a source to destination. There are mainly two categories of routing protocols in mobile ad-hoc network. First, on-demand(or reactive) protocols such as (DSR, AODV), in these protocols route is established by the nodes before sending the packet and also route is maintained by the node only when needed. Second, proactive protocols such as DSDV, where the nodes exchange routing information periodically and tries to have information about the current route to destination. Some of the major issues which we should take into consideration is FLOODING which is a straight forward approach in which a source node broadcast a packet to its one-hop neighbor which in turn rebroadcast to its one-hop neighbor i.e is sender's 2-hop neighbor until all nodes are reachable. This also have disadvantage of message contention and redundancy .The main cause of FLOODING is rebroadcast messages which is caused due to repeated broadcasting technigue.In this paper we have Proposed (MDSR) algorithm in reactive routing protocol in which we maintain the information of two-hop neighbour and MPR node in cache. This algorithm reduces the amount of control messages, thus further reduces the control overhead.

\section{RELATED WORK}

M.BaniYassein et al[4]. They have given the probabilistic approach to flooding with the aim of reducing rebroadcast thus alleviating broadcast storm problem. They have proposed a new probabilistic approach that dynamically adjust the rebroadcasting probability as per the node distribution. When nodes have low density then rebroadcast probability increases while nodes having high density then rebroadcast probability decreases. Simulation results have shown that this algorithm reduce rebroadcast upto $50 \%$ without affecting other factors .The major drawback is that always a threshold value must be maintained to decide the value of rebroadcast probability.

Jae-sooKim et al[8].Authors have proposed a dynamic broadcasting method in combination of area based and neighbor confirmation for MANET. Mobile host dynamically adjust rebroadcast probability according to coverage area of neighbor which is estimated by the distance from sender Simulation results compared to flooding, it has fewer rebroadcast messages. It also provides higher reachability and low collision. The problem with this proposal is always we have to decide a sensitivity value for rebroadcast probability.

R.Balakrishna et al[1].Authors have proposed this algorithm to reduce number of forwarding nodes. They have proposed a simple algorithm as double-coverage broadcast[1]which selects forwarding nodes from sender's 1-hop neighbor in greedy manner and thus it forwards the packets further and send acknowledgement to sender and the non forwarding node does not forwards the packets or no acknowledgement is send to sender. This proposal avoids broadcast storm problem by reducing rebroadcast messages.

WARDI et al[3].In this paper, authors have presented REOLSR, a modification MPR selection mechanism of OLSR protocol. The REOLSR selects nodes from one hope neighbor based on residual energy. Thus, the nodes with higher remaining energy are considered as MPR and avoid selecting nodes with lower residual energy. The performance of the REOLSR was examined through NS2.34 with UM-OLSR. The simulation results show that REOLSR achieve better throughput when compared with the MPR selection standard .The main drawback is every time calculating the energy for each node becomes complex.

PENG Wei et al [9]In this authors have presented a. new approach for efficient broadcasting in mobile ad hoc network in which each node maintains two data structures: a duplicate table and a two-hop neighbor table .Entries of the duplicate table which checks whether the broadcast message received is duplicate or not and second is 2-hop neighbor table maintains topology information of network. The proposed protocol called AHBP selects BRG from the senders'1-hop neighbor in greedy manner. It can also be taken as connected dominating set .Only BRG's can rebroadcast message other's are only broadcast receivers. The protocol can reduce redundancy and broad-cast storm problem to some extent and also saves network's 
bandwidth. The major drawback of this protocol is that it requires extra overhead to calculate BRG thus also becomes complex.

\section{ROUTING MECHANISM OF DSR}

The Dynamic Source Routing protocol (DSR) is a simple routing protocol use in multi-hop wireless ad hoc networks .It is different from other's that it uses source routing supplied by packet's originator to determine packet's path through the network .In this packets carries complete ordered list of nodes in its header through which the packet will pass. The protocol is composed of the two mechanisms of Route Discovery and Route Maintenance, to allow nodes to discover and maintain source routes to arbitrary destinations in the ad hoc network .In this routes are kept in route cache, which further helps in minimizing route discovery and also message redundancy.

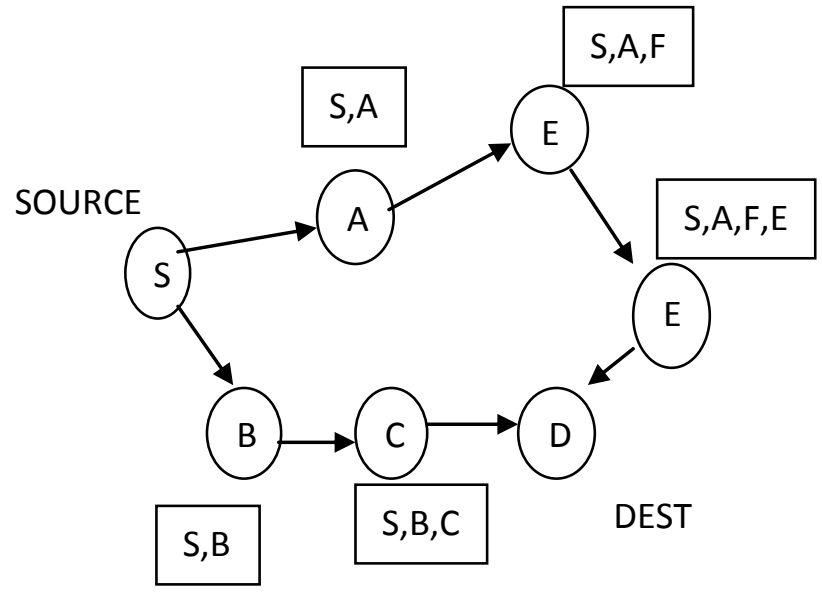

Fig-1 RREQ in DSR

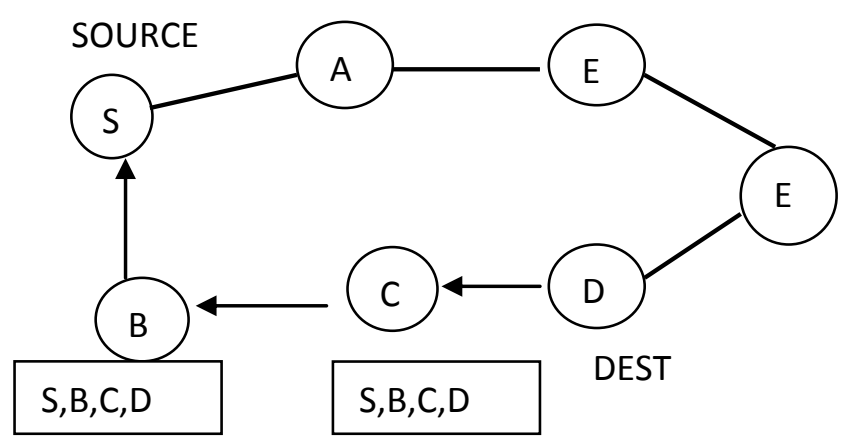

Fig-2 RREP in DSR

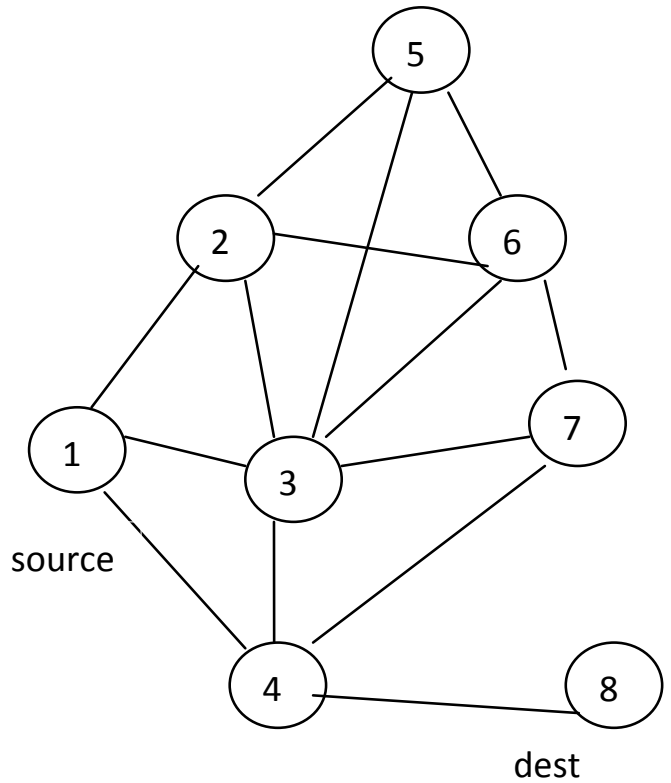

Fig- 3 source routing in DSR

Table-1 Total Msg Using DSR

\begin{tabular}{|l|}
\hline Total Messages \\
\hline $1=2$ \\
\hline $3=4$ \\
\hline $4=4$ \\
\hline $5=3$ \\
\hline $6=5$ \\
\hline $7=4$ \\
\hline $8=2$ \\
\hline total $=31$ \\
\hline
\end{tabular}

\section{M-DSR REACTIVE ROUTING}

\section{ALGORITHM}

.Each node in the network selects from its one-hop neighbor nodes, called multipoint relays (MPRs), as the forwarding node set to retransmit broadcast packets. Other nodes that are not in the MPR set can read but not retransmit broadcast packets. The MPR set guarantees that all two-hop neighbor nodes of each node receive a copy of the broadcast packets. 


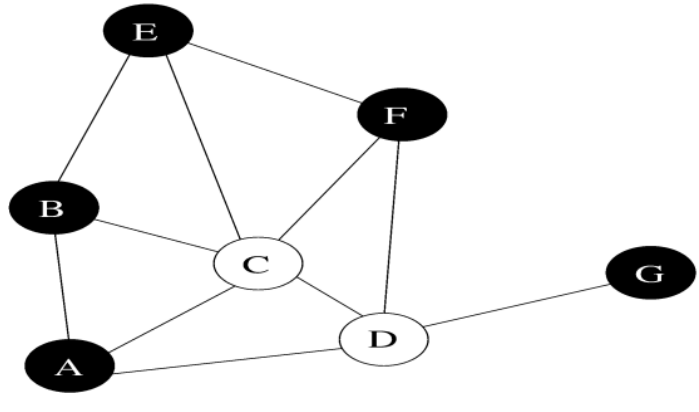

Fig-4 $C$ and D are MPR set

\subsection{Algorithm 1: MPR selection}

Input : A node , p's 1-hop neighborhood is N1 and p's 2-hop neighborhood is $\mathrm{N} 2$

Output :Mp, i.e. the MPR set of $\mathrm{p}$.

1. Begin

2. Add to Mp the nodes in N1 which are the only

nodes to provide reachability to a node in $\mathrm{N} 2$ or the nodes with degree one.

3. Remove the nodes from N2 which are now

covered by a node in $\mathrm{Mp}$.

4. while $\mathrm{N} 2 \neq \varnothing$ do

5. For each node in $\mathrm{N} 1$, calculate the reachability, i.e., the number of nodes in $\mathrm{N} 2$ that it can reach.

6. Add to $\mathrm{Mp}$ the node that provides the highest reachability. In case of multiple possibilities, select the node that has the highest degree.

7. Remove the nodes from $\mathrm{N} 2$ that are now covered by a node in $\mathrm{Mp}$.

8. End

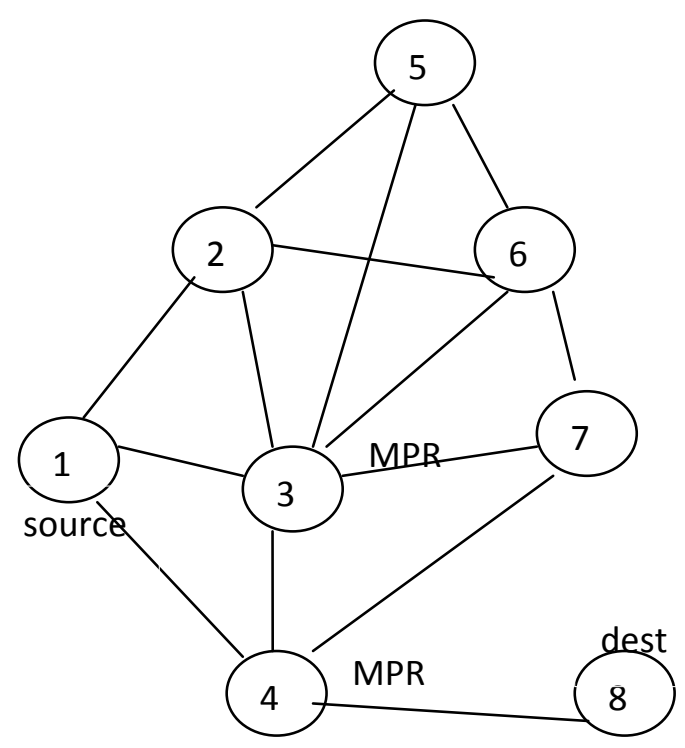

Fig-5 Example of MPR selection
Table-2 Two hop neighbor table

\begin{tabular}{|l|l|}
\hline 1 hop & 2 hop \\
\hline $1>2,3,4$ & $1>2>3,5,6$ \\
\hline $2>5,6$ & $1>3>2,4,5,6,7$ \\
\hline $3>5,6,7$ & $1>4>3,7,8$ \\
\hline $4>7,8$ & \\
\hline
\end{tabular}

\subsection{Message format}

- Hello msg \{source add, neighbours add\}

- Broadcast msg format \{originator's address , hop count $\}$

- Route message format \{SA,DA,MPR NODE\}

\subsection{Updation of cache in DSR routing protocol}

- 1-hop table

- 2-hop table

- MPR TABLE(node's add which is selected as MPR)

- MPR2 TABLE (Node's add which has selected the node as MPR)

Table-3 BROADCAST Message

\begin{tabular}{|c|c|c|}
\hline TYPE & LENGTH & HOPCOUNT \\
\hline \multicolumn{2}{|c|}{ SA } \\
\hline \multicolumn{2}{|c|}{ ADD OF HOP1 } \\
ADD OF HOP N \\
\hline
\end{tabular}

Table 4 M-RREQ Message

\begin{tabular}{|c|c|c|}
\hline TYPE & LENGTH RESV \\
\hline SA \\
\hline DA \\
\hline ADD OF MPR 1 \\
\hline$\ldots \ldots \ldots \ldots \ldots$ \\
ADD OF MPR N \\
\hline
\end{tabular}


Table 5 Comparative Analysis

\begin{tabular}{|l|l|}
\hline $\begin{array}{l}\text { Total packets } \\
\text { using DSR }\end{array}$ & Total packets using MPR \\
\hline $1=2$ & $1=2$ \\
\hline $2=4$ & $2=2$ \\
\hline $3=6$ & $4=2$ \\
\hline $4=4$ & $5=1$ \\
\hline $5=3$ & $6=1$ \\
\hline $6=6$ & $7=2$ \\
\hline $7=4$ & $8=1$ \\
\hline $8=2$ & Total $=13$ \\
\hline Total $=31$ & \\
\hline
\end{tabular}

\section{CONCLUSION}

The paper discusses the existing DSR routing protocol and also the MPR selection mechanism. MPR selection mechanism in DSR routing protocol have been used to enhance broadcast storm problem. In MANETS there are various overhead problem in terms of flooding and broadcasting. The above mentioned problem has been catered by modifying the data structure of route request message and have included MPR field. In this paper the new proposed M-DSR routing algorith helps in minimizing the control overhead in reactive routing protocol .In this M-DSR data structure of message have been taken i.e BROADCAST MSG and M-RREQ MSG, that will further limit the no of control messages in the network which will increase the protocol performance .The proposed algorithm helps to reduce the no of packets in DSR routing protocol and also reduce the rebroadcast messages. It reduces the total overhead in the network. Further It also helps to reduce the broadcast storm problem. Thus by reducing the rebroadcast messages it can also reduce the problem of contention. In our future work we are working for implementation of this algorithm in ns2 and compare its performance with other routing protocol.

\section{REFERENCES}

[1] R.Balakrishna,U.RajeswarRao,G.A.Ramachandra."Reliabil ity in MANET"s Using Enhanced Double Coverage
Broadcasting"Int.J. of Advanced Networking and ApplicationsVolume:01 Issue:03 Page:147 153(2009)

[2] Changling Liu, Jörg Kaiser , "A Survey of Mobile Ad Hoc network Routing Protocols", the University of Magdeburg , October 2005

[3] WARDI,Kouji,HIRATA,YoshinobuHIGAMI, "Energy Aware MPR Selection Mechanism in OLSR basedMobile Ad Hoc Networks" GRADUATE SCHOOL OF SCIENCE AND ENGINEERING, EHIME UNIVERSITY3 Bunkyo-Cho Street, Matsuyama, Ehime, 790-8577 Japan, PAK vol. 56, nr 11/2010

[4] Chandra .R, V. Ramasubramanian, and K.P. Birman, "Anonymous gossip: Improving mul-ticast reliability in mobile ad-hoc networks," Proc. of IEEE Int'l conf. on Distributed Com-puter System (ICDCS'2001), pp. 275283, Apr. 2001.

[5] Q. Zhang, D.P. Agrawal , Dynamicprobabilistic broadcasting in MANETs, J.Parallel Distrib. Comput. Vol 65, pp. 220-233, 2005.

[6] Peng.W and X.Lu,"An efficient broadcast protocol for mobile ad hoc networks,"Journal of Science and Technology,Beijing,china,2002

[7] B.Williams and T. Camp, "Comparison of Broadcasting Techniques for Mobile Ad Hoc Networks,'Proc.of3rdACMInt'1SymposiumonMobileAdH ocNetworkingandComputing(MOBIHOC'2002),pp.194205,Jun.2002

[8] Jae-soo Kim"Probabilistic Broadcasting Based on Coverage Area and Neighbor Confirmation in Mobile Ad Hoc Networks"IEEE Communications SocietyGlobecom 2004 Workshops

[9] 9 .PENG Wei and LU Xichengand LU Xicheng. "AHBP: An Efficient Broadcast Protocol for Mobile Ad Hoc Networks" Department of Computer Science, Changsha Institute of Technology, Vol.16 No.2 March 2001

[10] G. Allard, P. Jacquet, and L. Viennot, "Ad Hoc Routing Protocolswith Multipoint Relaying," 5eme Rencontres Francophones sur les aspects Algorithmiques des Telecommunications, (Algo-Tel'2003).

[11] J. Wu and F. Dai, "A Generic Distributed Broadcast Scheme inAd Hoc Wireless Networks," IEEE Trans. Computers, vol. 53,Oct. 2004, pp. 1343-54. 